\title{
Une nouvelle caractérisation des sphères géodésiques dans les espaces modèles
}

\author{
Julien Roth ${ }^{\mathrm{a}}$, \\ ${ }^{a}$ Laboratoire d'Analyse et de Mathématiques Appliquées, Université Paris-Est, 5 \\ boulevard Descartes-Champs-sur-Marne, 77454 Marne-la-Vallée Cedex 2 \\ Reçu le ${ }^{* * * * *}$; accepté après révision le +++++ \\ Présenté par
}

\section{Résumé}

Nous montrons qu'une hypersurface compacte, immergée dans l'espace hyperbolique ou l'hémisphère ouvert, de courbure moyenne constante et dont la courbure scalaire est presque constante est une sphère géodésique. Pour citer cet article : A. Nom1, A. Nom2, C. R. Acad. Sci. Paris, Ser. I 340 (2005).

\begin{abstract}
A new characterization of geodesic spheres in space forms. We show that a compact immersed hypersurface of hyperbolic space or an open half-sphere with constant mean curvature and almost constant scalar curvature is a geodesic sphere.

To cite this article: A. Nom1, A. Nom2, C. R. Acad. Sci. Paris, Ser. I 340 (2005).
\end{abstract}

\section{Abridged English version}

The well-Known Alexandrov theorem [1] claims that any connected, oriented, compact hypersurface without boundary embedded into the Euclidean space $\mathbb{R}^{n+1}$ with constant mean curvature (CMC) is a geodesic sphere. The assumption that the hypersurface is embedded is crucial. Indeed, the result is false for immersed hypersurfaces. For instance, the so-called Wente's tori (see [11]) are examples of (non-embedded) immersed surfaces in $\mathbb{R}^{3}$ with constant mean curvature which are not a geodesic spheres. Moreover, examples of compact CMC surfaces with higher genus are known $[7,6]$.

Email address: julien.roth@univ-mlv.fr (Julien Roth). 
Always for surfaces in $\mathbb{R}^{3}$, Hopf [3] proved that CMC immersed spheres are geodesic spheres. Here, the result is not true in any dimension since Hsiang, Teng and $\mathrm{Yu}$ have constructed examples of CMC spheres in higher dimension which are not geodesic spheres [5].

Note that the Alexandrov Theorem is still valid for hypersurfaces of the hyperbolic space or the open half-sphere. Indeed, with have the following statement due to Montiel and Ros [9], a compact hypersphere embedded into the hyperbolic space or the open half-sphere with constant mean curvature is a geodesic hypersphere.

In [10], we find an alternative assumption to the embedding such that under this assumption, CMC hypersurfaces are geodesic spheres. Namely, we have proved that a CMC hypersurface of $\mathbb{R}^{n}$ with almost constant scalar curvature is a geodesic sphere and then the scalar curvature is constant. The aim of this note is to prove that this result is still valid in the hyperbolic space or the half-sphere. We show the following

Theorem 0.1 Let $\left(M^{n}, g\right)$ be a compact without boundary, connected and oriented Riemannian manifold, isometrically immersed into the simply connected space form $\mathbb{M}_{\delta}^{n+1}$ of constant sectional curvature $\delta$. If $\delta>0$, we assume that $M$ is contained in a ball of radius $\frac{\pi}{4 \sqrt{\delta}}$. Let $h>0$ Then, there exists $\varepsilon(n, h, \delta)>0$ such that if $M$ has constant mean curvature $H=h$, and $|S c a l-s|<\varepsilon$ for a constant s, then $M$ is a geodesic sphere and its scalar curvature is constant, equal to $n(n-1)\left(h^{2}+\delta\right)$.

The proof of this statement relies on an eigenvalue pinching result due to Grosjean [2].

\section{Introduction}

Le théorème d'Alexandrov [1] assure qu'une hypersurface connexe, compacte sans bord, orientée, plongée isométriquement dans l'espace euclidien à courbure moyenne constante $(\mathrm{CMC})$ est une sphère géodésique. L'hypothèse de plongement est nécessaire. En effet, il existe d'autres surfaces compactes CMC immergées dans $\mathbb{R}^{3}$, comme par exemple, les tores de Wente [11]. Récemment, Jleli et Pacard [6] ont construit des exemples de genre arbitraire. Toujours dans le cas des surfaces de $\mathbb{R}^{3}$, Hopf [3] a montré qu'une sphère CMC immergée est une sphère géodésique (et donc plongée). Les exemples de sphères CMC immergées non plongées construits par Hsiang, Teng et $\mathrm{Wu}$ [5] en dimension supérieure montrent que le théorème de Hopf n'est pas vrai en toute dimension.

Notons également que le théorème d'Alexandrov est valable également dans l'espace hyperbolique et l'hémisphère ouvert. En effet, Montiel et Ros [9] ont montré qu'une hypersurface compacte plongée dans l'espace hyperbolique et 
l'hémisphère ouvert est une hypersphère géodésique.

Dans [10], nous avons donné un résultat de ce type pour les hypersurfaces de $\mathbb{R}^{n}$ pour tout $n \geq 3$ en remplaçant l'hypothèse de plongement (ou d'être topologiquement une sphère) par une hypothèse métrique. Précisément, une hypersurface compacte $\mathrm{CMC}$ dont la courbure scalaire est presque constante est une sphère géodésique, et donc sa courbure scalaire est constante et elle est plongée.

Le but de cette note est de montrer que ce résultat est également vrai pour les hypersurfaces de l'espace hyperbolique et de l'hémisphère ouvert.

Avant d'énoncer ce résultat, nous introduisons la notation suivante : $\mathbb{M}_{\delta}^{n}$ est l'espace euclidien si $\delta=0$, l'espace hyperbolique de courbure sectionnelle $\delta$ si $\delta<0$ et enfin l'hémispère ouvert de courbure sectionnelle $\delta$ si $\delta>0$. Si $\delta>0$, on supposera que $M$ est contenue dans une boule de rayon $\frac{\pi}{4 \sqrt{\delta}}$. Le résultat est le suivant :

Théorème 1.1 Soit $h>0$, il existe une constante $\varepsilon(n, \delta, h)>0$ dépendant uniquement de $n, \delta$ et $h$ telle que si $\left(M^{n}, g\right)$ est une variété riemannienne connexe compacte immergée isométriquement dans $\mathbb{M}_{\delta}^{n+1}$ (si $\delta>0$, on suppose $M$ est contenue dans une boule de rayon $\frac{\pi}{4 \sqrt{\delta}}$ ) avec courbure moyenne constante égale à $h$ et $|S c a l-s| \leq \varepsilon$, alors $M$ est une sphère géodésique et donc $M$ est plongée et sa courbure scalaire est constante égale à $n(n-1)\left(h^{2}+\delta\right)$.

Nous avons également ce second théorème où les rôles des courbures moyenne et scalaire sont inversés.

Théorème 1.2 Soit $s>0$, il existe une constante $\varepsilon^{\prime}(n, \delta, s)>0$ dépendant uniquement de $n, \delta$ et $s$ telle que si $\left(M^{n}, g\right)$ est une variété riemannienne connexe compacte immergée isométriquement dans $\mathbb{M}_{\delta}^{n+1}$ (si $\delta>0$, on suppose $M$ est contenue dans une boule de rayon $\frac{\pi}{4 \sqrt{\delta}}$ ) avec courbure scalaire constante égale à s et $|H-h| \leq \varepsilon^{\prime}$, alors $M$ est une sphère géodésique et donc $M$ est plongée et sa courbure moyenne est constante égale à $\sqrt{\frac{s}{n(n-1)}-\delta}$.

\section{Préliminaires}

Avant de donner la preuve des Théorèmes 1.1 et 1.2, nous rappelons quelques résultats préliminaires. Tout d'abord, nous introduisons les fonctions suivantes :

$$
s_{\delta}(t)=\left\{\begin{array}{lr}
\frac{1}{\sqrt{\delta}} \sin (\sqrt{\delta} t) & \text { si } \delta>0 \\
\frac{1}{\sqrt{-\delta}} \sinh (\sqrt{-\delta} t) & \text { si } \delta<0
\end{array} \quad \text { et } \quad c_{\delta}(t)= \begin{cases}\cos (\sqrt{\delta} t) & \text { si } \delta>0 \\
\cosh (\sqrt{-\delta} t) & \text { si } \delta<0\end{cases}\right.
$$

Considérons un point $p_{0} \in \mathbb{M}_{\delta}^{n+1}$, on note $r(x)=d\left(p_{0}, x\right)$ la distance au point $p_{0}$. Pour une hypersurface $M$ dans $\mathbb{M}_{\delta}^{n+1}$, nous avons les formules suivantes, 
dites de Hsiung-Minkowski, pour $k \in\{0, \cdots, n-1\}$ :

$$
\int_{M}\left(c_{\delta}(r) H_{k}+s_{\delta}(r)<\bar{\nabla} r, \nu>\right) d v_{g}=0,
$$

où $H_{k}$ est la $k$-ième courbure moyenne, c'est-à-dire, le $k$-ième polynôme symétrique des courbures principales, $\nu$ le champ normal unitaire et $\bar{\nabla} r$ le gradient ambiant de la fonction distance à $p_{0}$. Par convention, $H_{0}=1$, et il est clair que $H_{1}$ est la courbure moyenne $H$. D'autre part, la formule de Gauss implique que $H_{2}=\frac{s}{n\left(n_{1}\right)}-\delta$.

Nous rappelons la formule de Gauss, ici dans sa version tracée faisant intervenir la courbure de Ricci :

$$
\operatorname{Ric}(Y)=\overline{\operatorname{Ric}}(Y)-\bar{R}(\nu, Y, \nu, Y)+n H\langle A Y, Y\rangle-\left\langle A^{2} Y, Y\right\rangle,
$$

où $A$ est la seconde forme fondamentale.En traçant une seconde fois, on obtient :

$$
S c a l=n(n-1) \delta+n^{2} H^{2}-|A|^{2},
$$

Enfin, nous finissons cette section de préliminaires en rappelant le résutlat suvant :

Théorème 2.1 (Grosjean, [2]) Soit $M$ une hypersurface connexe compacte orientée de $\mathbb{M}_{\delta}^{n+1}$. Si $\delta>0$, on suppose que $M$ est contenue dans une boule de $\mathbb{M}_{\delta}^{n+1}$ de rayon $\frac{\pi}{4 \sqrt{\delta}}$. Soit $\left.\theta \in\right] 0,1\left[\right.$, alors il existe une constante $C_{\theta}\left(n,\|B\|_{\infty}, V(M), \delta\right)>$ 0 telle que si

$$
n\left(\|H\|_{\infty}+\delta\right)-C_{\theta}<\lambda_{1}(\Delta),
$$

alors $M$ est difféomorphe et $\theta$-quasi-isometrique à une sphère géodésique de rayon $\sqrt{\frac{n}{\lambda_{1}}}$.

\section{Preuve des Théorèmes 1.1 et 1.2}

Les Théorèmes 1.1 et 1.2 sont une conséquence du résultat suivant

Lemme 3.1 Soit $\left(M^{n}, g\right)$ une variété riemannienne connexe, orientée, compacte sans bord, immergée isométriquement dans $\mathbb{M}_{\delta}^{n+1}$. Soient $s, h>0$ tel que $|H-h|<\varepsilon$ et $|S c a l-s|<\varepsilon$, alors $\left|h^{2}+\delta-\frac{s}{n(n-1)}\right|<A(n, \delta, h) \varepsilon$.

Remarque 1 Ce Lemme est une version améliorée du lemme que nous prouvions dans [10]. En effet, ici, la constante $A$ ne dépend pas du rayon extrinsèque. Notons également que si $\delta>0$ nous n'avons pas besoin de supposer 
que $M$ est contenue dans une boule de rayon $\frac{\pi}{\sqrt{\delta}}$. Cette hypothèse seulement nécessaire par la suite pour appliquer le théorème de Grosjean.

Preuve : Il est facile de voir qu'à partir des formules de Hsiung-Minkowski, nous obtenons :

$$
\left(h-\frac{h_{2}}{h}\right) \int_{M} c_{\delta}(r)+\frac{h_{2}}{h} \int_{M}(h-H)\langle Z, \nu\rangle+\int_{M} c_{\delta}(r)(H-h)+\int_{M}\left(H_{2}-h_{2}\right)\langle Z, \nu\rangle=0
$$

où $Z=s_{\delta}(r) \bar{\nabla} r$, ce qui donne avec le fait que $|Z|=s_{\delta}(r)$ :

$$
\left|h-\frac{h_{2}}{h}\right| \int_{M} c_{\delta}(r) \leqslant \frac{h_{2}}{h} \varepsilon \int_{M} s_{\delta}(r)+\varepsilon \int_{M} c_{\delta}(r)+\frac{\varepsilon}{n(n-1)} \int_{M} s_{\delta}(r)
$$

De plus, comme $\left|H_{2}\right| \leqslant H^{2}$, on déduit $\left|h_{2}\right| \leqslant h^{2}+A_{1}(n, h, \delta) \varepsilon$, et ainsi

$$
\left|h-\frac{h_{2}}{h}\right| \leqslant \varepsilon+A_{2}(n, h, \delta) \varepsilon \frac{\int_{M} s_{\delta}(r)}{\int_{M} c_{\delta}(r)}
$$

Enfin, on voit facilement que comme $\delta \neq 0, \frac{\int_{M} s_{\delta}(r)}{\int_{M} c_{\delta}(r)}<\frac{1}{4 \sqrt{\delta}}$. En remplaçant dans l'inégalité précédente, on obtient le résultat souhaité.

Nous pouvons maintenant finir la preuve. En utilisant ce lemme et la formule de Gauss (1), on obtient alors que l'hypersurface est presque ombilique, et par conséquent presque Einstein

$$
\left.\left|\operatorname{Ric}(Y)-(n-1)\left(h^{2}+\delta\right)\right| Y\right|^{2} \mid \leqslant A_{3}(n, h, \delta) \varepsilon .
$$

L'inégalité de Lichnerowicz [8] permet d'obtenir une minoration de la première valeur propre du laplacien sur $M$

$$
\lambda_{1}(\Delta) \geqslant n\left(h^{2}+\delta\right)-o(\varepsilon)
$$

Or cette minoration donne exactement l'hypothèse de pincement du Théorème 2.1. En choisissant $\varepsilon$ suffisamment petit, on conclut que $M$ est difféomorphe à une sphère géodésique de $\mathbb{M}_{\delta}^{n+1}$. De plus, ce difféomorphisme est de la forme $F=G \circ \Phi$, où $\Phi$ est l'immersion de $M$ dans $\mathbb{M}_{\delta}^{n+1}$ (voir les détails dans [2]). Or, $F$ ne peut être bijective que si l'immersion $\Phi$ est injective. De ce fait, $\Phi$ est un plongement et le théorème d'Alexandrov dans $\mathbb{M}_{\delta}^{n+1}$ implique que $M$ est donc une sphère géodésique.

Notons enfin que $\varepsilon$ ne dépend que de $n, h$ et $\delta$. Tout d'abord, Comme la courbure scalaire est presque constante, la formule de Gauss (2) permet de 
remplacer la dépendance en $\|A\|_{\infty}$ par une dépendance en $h$. Enfin, le volume est entièrement contrôlé par $n, h$ et $\delta$. En effet, comme la courbure de Ricci est minorée par l'inégalité (3), le volume est majoré grâce au théorème de Bishop. D'autre part le volume est minoré en fonction de $n, h$ et $\delta$. Précisément, nous avons $\operatorname{Vol}(M) \geq \frac{\alpha(n, \delta)}{h^{n}}$ grâce à l'inégalité de Sobolev extrinsèque de Hoffman et Spruck [4],

$$
\left(\int_{M} f^{\frac{n}{n-1}} d v_{g}\right)^{\frac{n-1}{n}} \leq K(n, \delta) \int_{M}(|d f|+|H| f) d v_{g},
$$

en prenant $f \equiv 1$. Ceci achève la preuve du théorème 1.1 .

La preuve du second théorème est analogue, on conclut en utilisant le théorème d'Alexandrov pour la courbure scalaire de Montiel et Ros [9].

\section{Références}

[1] A.D. Alexandrov, A characteristic property of spheres, Ann. Mat. Pura Appl., 58 (1962), 303-315.

[2] J.F. Grosejan, Eigenvalue pinching and application to the stability and the almost umbilicity of hypersurfaces, arxiv :0709.0831v2 .

[3] H. Hopf Differential Geometry in the large, Lecture Notes in Mathematics, 1000, (Springer-Verlag, 1983).

[4] D. Hoffman and J. Spruck, Sobolev and isoperimetric inequalities for Riemannian submanifolds, Comm. Pure. and Appl. Math., 28, (1975), 765-766.

[5] W. Hsiang, Z. Teng and W. Yu, New examples of constant mean curvature immersions of (2k-1)-spheres into Euclidean 2k-space, Ann. Math., 117(3), 609-625 .

[6] M. Jleli and F. Pacard, An end-to-end construction for compact constant mean curvature surfaces, Pac. J. Math., 221(1) (2005), 81-108

[7] N. Kapouleas, Constant mean curvature surfaces constructed by fusing Wente tori, Invent. Math. , 119(3), (1995), 443-518.

[8] A. Lichnerowicz, Géométrie des groupes de transformation, (Dunod, 1958).

[9] S. Montiel and A. Ros, Compact hypersurfaces : the Alexandrov theorem for higher order mean curvature, Pitman Monographs Surveys Pure Appl. Math. 52, (1991), 279-296.

[10] J. Roth, Rigidity results for geodesic spheres in space forms, Differential Geometry, Proceedings of the VIII International Colloquium, (World Scientific, 2009), 156-163.

[11] H. Wente, Counterexample to a conjecture of H. Hopf, Pac. J. Math., 121(1) (1986), 193-243. 